Review began 02/23/2022 Review ended 02/28/2022 Published 03/06/2022

๑) Copyright 2022 Giri et al. This is an open access article distributed under the terms of the Creative Commons Attribution License CC-BY 4.0., which permits unrestricted use, distribution, and reproduction in any medium, provided the original author and source are credited.

\section{Impact of Treatment Supporters on the Treatment Outcomes of Drug Resistant- Tuberculosis (DR-TB) Patients: A Retrospective Cohort Study}

\author{
Om P. Giri ${ }^{1}$, Abhay Kumar ${ }^{2}$, Vishal P. Giri ${ }^{3}$, Nishant Nikhil ${ }^{4}$ \\ 1. Pulmonary Medicine, Darbhanga Medical College, Darbhanga, IND 2. ENT, Uttar Pradesh University of Medical \\ Sciences, Saifai, IND 3. Pharmacology, Autonomous State Medical College, Shahjahanpur, IND 4. Drug Resistant- \\ Tuberculosis (DR-TB) Centre, Darbhanga Medical College, Darbhanga, IND
}

Corresponding author: Vishal P. Giri, drvpgiri@gmail.com

\begin{abstract}
Background: Drug resistant-tuberculosis (DR-TB) patients are provided universal drug susceptibility testing (UDST), anti-TB drugs for the treatment of DR-TB, nutritional support (Nikshay Poshan Yojana - the financial incentive of rupees five hundred per month for each notified DR-TB patient for the duration for which the patient is on anti-TB drugs) by the Government of India.
\end{abstract}

Methods: This retrospective cohort record-based study was conducted in DR-TB patients. Some 1095 DR-TB patients who have initiated treatment at Nodal DR-TB Centre, Darbhanga Medical College and Hospital (DMCH), Darbhanga, and continued their anti-TB drugs at home blocks were followed till their treatment outcome was known. Data were analyzed by statistical experts of DMCH.

Results: Treatment supporters comprised 688 (62.83\%) females and 407 (37.17\%) males. Different types of treatment supporters noted were accredited social health activists (ASHAs) 622 (56.80\%), family members 365 (33.33\%), and community health workers 108 (09.86\%). Treatment outcome as transfer out was observed in 08 (1.29\%), 10 (2.74\%), and 13 (12.03\%) cases among ASHAs, family members, and community health workers, respectively [statistically significant $(\mathrm{p}<0.0001)]$.

Conclusion: ASHAs proved to be the best treatment supporters in comparison to both family members and community health workers for multi-drug resistant TB (MDR/RR-TB) patients.

Categories: Pulmonology, Public Health, Therapeutics

Keywords: multi-drug resistant tb -mdr, drug-resistant tuberculosis, tuberculosis treatment supporters, treatment outcome, dr-tb

\section{Introduction}

Globally about half a million new cases of rifampicin resistant tuberculosis (RR-TB) occurred in 2019, out of which 78\% had multi-drug resistant TB (MDR-TB). The estimated number of MDR/RR-TB cases in India is 124000 (9.1 per lakh population).

The DR-TB cases have been classified as (a) RR-TB (a TB patient, whose biological specimen is resistant to rifampicin); (b) MDR-TB (a TB patient, whose biological specimen is resistant to both isoniazid and rifampicin); (c) pre-extensively drug resistant (XDR)-TB (an MDR-TB patient with additional resistance to fluoroquinolone; (d) XDR-TB (pre-XDR-TB patient with additional resistance to either bedaquiline (Bdq) or linezolid (or both); and (e) H mono/poly DR-TB (a TB patient, whose biological specimen is sensitive to rifampicin but resistant to isoniazid).

At present the various DR-TB treatment regimens used for the management of DR-TB cases are (a) shorter oral Bdq-containing MDR/RR-TB regimen: it consists of intensive phase (IP) of [(4-6) Bdq (6), levofloxacin (Lfx), clofazimine (Cfz), Z, E, H-h, ethionamide (Eto)] and continuation phase (CP) of [5 (Lfx, Cfz, Z, E)], total duration is of 9-11 months ; (b) longer oral M/XDR-TB regimen is of 18-20 months with no IP and CP, [1820 Lfx, Bdq (six months or longer), linezolid (Lzd), Cfz, cycloserine (Cs)]; (c) bedaquiline, pretomanid, and linezolid (BPaL) regimen [(6-9 months) BPaL] and (d) H mono/ poly DR-TB regimen [(six or nine months) Lfx, R, E, Z].

Shorter oral Bdq containing MDR/ RR-TB regimen: inclusion criteria are (a) MDR/ RR-TB with $\mathrm{H}$ resistance (INA/ KatG mutation only, not both), (b) MDR/ RR-TB with fluoroquinlone (FQ) resistance not detected, (c) children (aged 5 to less than 18 years age) and weighing at least $15 \mathrm{~kg}$, (d) no history of exposure to Bdq, Lfx, ethionamide (Eto) or Cfz for more than one month, (e) no bilateral cavitatory disease/ extensive 
parenchymal damage, on chest radiography), (f) no military TB, TB meningitis, CNS-TB, (g) women who are not pregnant or lactating, (h) no uncontrolled cardiac arrhythmia, (i) QTcF interval less than $500 \mathrm{~ms}$ in electrocardiogram (ECG), (j) normal serum electrolytes, and (k) no history of additional risk factors for torsades de pointes. All the above criteria must be met.

Longer oral M/XDR-TB regimen: inclusion criteria are (a) MDR/ RR-TB patients who are excluded from shorter oral Bdq-containing regimen, (b) XDR-TB patients, and (c) additional resistance/intolerance/ nonavailability of any drug used in shorter oral Bdq-containing regimen. Bedaquiline, pretomanid, and linezolid (BPaL) regimen is used in MDR-TB with additional resistance to FQ (pre-XDR-TB). H mono/ poly DR-TB regimen is used in $\mathrm{H}$ mono/poly DR-TB cases.

Stake holders of DR-TB services in local health system are physician at N/ DDR TBC, health facility doctor, DTO (District TB officer)/CDO (communicable disease officer), STS (senior treatment supervisor), STLS (senior TB laboratory supervisor), senior DR-TB co-ordinator, counselor at NDR-TBC, and treatment supporter. Each has a distinct function. Treatment supporter takes ambulatory care of the patient (counseling, linkages, support, and monitoring) [1].

The main role of a treatment supporter is to ensure that the patient takes the anti-TB drugs regularly, on schedule, for the full duration of treatment. The other roles of a treatment supporter are -- (a) to listen to the patient and encourage the patient to ask the questions about things that might be difficult to understand, (b) agree on a time and place to meet with the TB patient, (c) give the patient anti-TB drugs at each appointment according to the schedule, (d) record on the TB treatment card each time patient takes the drugs, and (e) refer the patient to the health facility when needed [2].

A treatment supporter can be any personnel from the medical officer, ANM (auxiliary nurse midwife), ASHA (accredited social health activist), CHW (community health worker), family member, and worker from the private sector (Project JEET). As far as possible only non-govt salaried personnel should be assigned as a treatment supporter to the patient. In exceptional circumstances, salaried National Tuberculosis Elimination Program (NTEP)/General Health System staff may also be assigned as a treatment supporter but they will not be eligible for any honorarium. Honorarium to the treatment supporter to be disbursed upon completion or cure of DR-TB patient is Rs. 5000 (five thousand). At the end of the intensive phase (IP) Rs. 2000 (Two thousand) or less - initiation date + six months has passed. At the end of the continuation phase (CP) Rs. 3000 (three thousand) or less -- if the patient has treatment outcome assigned as either "Cured" or "Treatment Completed" [3].

The present study aims to evaluate the impact of different types of TB treatment supporters on the final treatment outcomes of MDR/RR-TB patients.

\section{Materials And Methods}

\section{Study design}

The present study is a retrospective cohort study wherein, the details of the patients who underwent treatment were obtained from available hospital records. The institutional ethics committee approved the study and granted a waiver of the patient consent process.

\section{Inclusion criteria}

All MDR/RR-pulmonary TB patients, whose treatment was initiated at Nodal DR-TB Centre (NDR-TBC), Darbhanga Medical College \& Hospital (DMCH), Laheriasarai, Darbhanga during the period from 1st September 2016 to 31st December 2020 and sent back through CDO (DTO) Darbhanga to their respective primary health centers (PHCs) in Darbhanga District were studied till their final treatment outcome was known.

\section{Diagnosis and follow-up monitoring}

Microbiological investigations of these patients were done at the time of diagnosis and during follow-up at NTEP accredited Damien Foundation India Trust (DFIT) medical laboratory (DMCH Campus, Darbhanga) which is well equipped with a fluorescent microscope, CBNAAT/ True Nat, FL-LPA, SL-LPA, and MGIT Culture. Their clinical monitoring was done at PHCs and laboratory investigations at the time of initiation of therapy and during follow-up were done in DFIT and Department of Clinical Pathology, DMCH.

\section{Organizational structure}

Darbhanga district has 18 PHCs (Tardih, Singhwara, Gora Bauram, Alinagar, Benipur, Manigachhi, Ghanshyampur, Biraul, Darbhanga Sadar, Baheri, Bahadurpur, Keoti, Kusheshwar Ashthan Satighat, Hanuman Nagar, Jale, Kusheshwar Ashthan East, Hayaghat, and Kiratpur), one CDO, one DOTS-PLUS Supervisor, one STS, and three TB Health Visitor (TBHV). ASHA, community health workers, and family members of the patients were engaged as treatment supporters in the service of MDR/RR-TB patients. 


\section{Cureus}

\section{Treatment outcome criteria}

(a) Cured -- A DR-TB patient who completed treatment with evidence of bacteriological response and no evidence of treatment failure. (b) Treatment completed -- A DR-TB patient who completed treatment but whose treatment outcome does not meet the definition for cured or treatment failed. (c) Died -- A DR-TB patient who died during the course of treatment. (d) Treatment failed -- A DR-TB patient whose treatment regimen needs to be terminated or permanently changed to a new regimen option or treatment strategy. (e) Lost to follow-up -- A DR-TB patient whose treatment was interrupted for two consecutive months or more. (f) Transferred out -- A DR-TB patient who was transferred to another treatment unit.

\section{Statistical analysis}

Pearson Chi-square test of independence was used and significant cut value was 95\%.

\section{Results}

A total of 1095 MDR/ RR-TB patients were studied, out of which 690 (63.01\%) were males and 405 (36.99\%) were females. The minimum age of the patient was 7 years and the maximum age was 87 years. The age group of 21-40 years was the most affected [622 (56.80\%)], followed by 7-20 years [241 (22.01\%)]. The age group of 80-87 years was least affected [4 (0.36\%)] (Table 1).

\begin{tabular}{|c|c|c|c|}
\hline Age group (years) & Male $\quad \mathrm{n}(\%)$ & Female n (\%) & Total $\mathbf{n}(\%)$ \\
\hline 07-20 & 130 (53.94) & $111(46.06)$ & $241(22.01)$ \\
\hline $21-40$ & $400(64.30)$ & $222(35.69)$ & $622(56.80)$ \\
\hline $41-60$ & $123(72.35)$ & 47 (27.65) & $170(15.52)$ \\
\hline $61-80$ & $34(58.62)$ & $24(41.38)$ & $58(5.30)$ \\
\hline $80-87$ & $03(75.00)$ & $01(25.00)$ & $04(0.36)$ \\
\hline Total & 690 (63.01) & 405 (36.99) & 1095 (100.00) \\
\hline
\end{tabular}

TABLE 1: Age group and gender distribution among DR-TB patients.

DR-TB, Drug resistant-tuberculosis

Some 346 (31.60\%) patients were declared cured, while 202 (18.45\%) had treatment outcome as treatment completed. Thus treatment success (cured plus treatment completed) noted among MDR/ RR-TB patients was 548 (50.05\%). Treatment failure was noted in 175 (15.98\%) (Table 2).

\begin{tabular}{|c|c|c|c|c|c|c|c|}
\hline Year & $\begin{array}{l}\text { Total number } n \\
\text { (\%) }\end{array}$ & $\begin{array}{l}\text { Cured n } \\
\text { (\%) }\end{array}$ & $\begin{array}{l}\text { Treatment completed } \mathrm{n} \\
\text { (\%) }\end{array}$ & $\begin{array}{l}\text { Died n } \\
(\%)\end{array}$ & $\begin{array}{l}\text { Treatment failed } n \\
(\%)\end{array}$ & $\begin{array}{l}\text { Lost to follow-up n } \\
\text { (\%) }\end{array}$ & $\begin{array}{l}\text { Transferred out } \mathbf{n} \\
\text { (\%) }\end{array}$ \\
\hline 2016 & $51(100.00)$ & 20 (39.21) & $4(7.84)$ & $11(21.57)$ & $3(5.88)$ & $10(19.61)$ & $3(5.88)$ \\
\hline 2017 & 141 (100.00) & 62 (43.97) & 18 (12.76) & $31(21.98)$ & $9(6.38)$ & 13 (9.22) & $8(5.67)$ \\
\hline 2018 & 184 (100.00) & 71 (38.59) & $15(8.15)$ & $26(14.13)$ & $30(16.21)$ & $39(21.19)$ & $3(1.63)$ \\
\hline 2019 & $334(100.00)$ & $83(24.85)$ & $78(23.35)$ & $50(14.97)$ & $49(17.66)$ & $47(14.07)$ & $17(5.09)$ \\
\hline 2020 & $385(100.00)$ & $110(28.57)$ & $87(22.60)$ & $70(18.18)$ & 74 (19.21) & 44 (11.43) & -- \\
\hline Total & 1095 (100.00) & $\begin{array}{l}346 \\
(31.60)\end{array}$ & 202 (18.45) & $\begin{array}{l}188 \\
(17.17)\end{array}$ & $175(15.98)$ & $153(13.97)$ & $31(2.83)$ \\
\hline
\end{tabular}

\section{TABLE 2: Treatment outcomes of DR-TB patients.}

DR-TB, Drug resistant-tuberculosis 


\section{Cureus}

supporters belonged to the age group of $31-40$ years, while $32.51 \%$ had age $>40$ years. Some $71.23 \%$ of family members who acted as treatment supporters had age $>40$ years, while $59.97 \%$ of ASHAs belonged to the age group of 31-40 years (Table 3).

\begin{tabular}{|c|c|c|c|c|}
\hline \multirow{2}{*}{$\begin{array}{l}\text { Category } \\
\text { Gender }\end{array}$} & \multicolumn{4}{|c|}{ Type of treatment supporters } \\
\hline & Total n (\%) & ASHA n (\%) & Family member n (\%) & Community health worker $\mathrm{n}(\%)$ \\
\hline Male & $407(37.17)$ & 00 & $322(90.96)$ & $75(69.44)$ \\
\hline Female & $688(62.83)$ & $622(100.00)$ & $33(9.04)$ & $33(30.56)$ \\
\hline Total & 1095 & 622 & 365 & 108 \\
\hline \multicolumn{5}{|c|}{ Age group (years) } \\
\hline $18-30$ & 209 (19.09) & 155 (24.42) & 05 (1.37) & $49(45.32)$ \\
\hline $31-40$ & $530(48.40)$ & $373(59.97)$ & $100(27.40)$ & $57(52.78)$ \\
\hline$>40$ & 356 (32.51) & $94(15.11)$ & $260(71.23)$ & $02(1.85)$ \\
\hline Total & 1095 & 622 & 365 & 108 \\
\hline
\end{tabular}

\section{TABLE 3: Baseline characteristics of treatment supporters.}

The ASHAs were associated as treatment supporters with $33.28 \%$ of cases who had treatment outcome as cured. Some $30.68 \%$ and $25.00 \%$ cured cases had treatment supporters as family members and community health workers respectively. The MDR/ RR-TB cases whose treatment outcome was declared as treatment completed had ASHAs, family members, and community health workers as treatment supporters in $18.33 \%$, $19.73 \%$, and $14.81 \%$ cases respectively. Treatment failure was observed in $15.91 \%, 15.89 \%$, and $16.67 \%$ cases that were supported by ASHAs, family members, and community health workers, respectively. Death was recorded in $16.27 \%$ of cases in the family members group, $14.81 \%$ lost to follow-up cases were noted in the community health worker group (Table 4 ).

\begin{tabular}{|c|c|c|c|c|}
\hline Category & ASHA & Family member & Community health worker & Statistical indicator \\
\hline Cured n (\%) & 207 (33.28) & $112(30.68)$ & $27(25.00)$ & $x 2=3.13, d f=2, p=0.2$ \\
\hline Treatment completed n (\%) & $114(18.33)$ & $72(19.73)$ & $16(14.81)$ & $\mathrm{x} 2=2.4, \mathrm{df}=2, \mathrm{p}=0.28$ \\
\hline Died n (\%) & $107(17.20)$ & $63(17.26)$ & $18(16.67)$ & $x^{2}=0.02, d f=2, p=0.98$ \\
\hline Treatment failed n (\%) & $99(15.91)$ & $58(15.89)$ & $18(16.67)$ & $x^{2}=1.06, d f=2, p=0.58$ \\
\hline Lost to follow up n (\%) & $87(13.90)$ & $50(13.70)$ & $16(14.81)$ & $x^{2}=0.086, d f=2, p=0.95$ \\
\hline Transferred out n (\%) & $08(1.29)$ & $10(2.74)$ & $13(12.03)$ & $x^{2}=38.6, d f=2, p<0.0001$ \\
\hline Total & 622 & 365 & 108 & 1095 \\
\hline
\end{tabular}

\section{TABLE 4: DR-TB treatment outcomes in relation to different types of treatment supporters.}

DR-TB, drug resistant-tuberculosis; ASHA, accredited social health activists

Among DR-TB, the least transfer of patients was seen among ASHAs (1.29\%) followed by family members $(2.74 \%)$ and community health workers $(12.03 \%)$ and this was statistically significant $(\mathrm{p}<0.0001)$ (Table 4).

\section{Discussion}

The present study revealed that males comprised $63.01 \%$ and females $36.99 \%$ of DR-TB cases. Some $56.80 \%$ of the DR-TB cases were of young age (21-40 years). This result is in accordance with a study conducted in Lucknow, which also ascertains that the males (69.40\%) suffer from DR-TB more than females (30.60\%) and the age group most involved was 21-30 years. Males were predominant (54.00\%) but higher age group (25-64 years) as the most affected (74.00\%) has been recorded by another study conducted in the 
Netherlands. Female predominance (56.10\%) among DR-TB cases has been reported by Javaid et al., and this gender distribution is not in accordance with our study and few studies mentioned above as all of them have mentioned males predominance in DR-TB [4-6].

The present study revealed that a larger number (62.83\%) of treatment supporters comprised females as compared to the males (37.17\%). Female treatment supporters comprised 622 (56.80\%) ASHAs, 33 (3.01\%) family members, and 33 (3.01\%) community health workers. Most (48.40\%) treatment supporters belonged to the age group of 31-40 years, followed by (32.51\%) more than 40 years age group. Family members of more than 40 years and ASHAs of 31-40 years were preferred as treatment supporters.

Females predominance (57.82\%) as treatment supporters has been also reported by a retrospective cohort study in Pakistan. Females comprised $60.00 \%$ family supporters, $51.10 \%$ health facility supporters, and $37.20 \%$ community supporters but most (41.39\%) treatment supporters had different age groups (15-25 years) as compared to the age groups (31-40 years) noted in the present study. This study further revealed that family members were chosen as treatment supporters by $86.80 \%$ of patients, $7.63 \%$ of patients selected community-based health workers, and $5.56 \%$ of cases chose facility-based health workers. Thus, family members were noted to be the first choice as treatment supporters. Our study is not in accordance with this study report as the present study has revealed that family members were chosen by $33.33 \%$ of patients (second choice), $9.86 \%$ of cases selected community-based health workers, and $56.80 \%$ cases chose a facilitybased health worker as treatment supporters [7].

Family members have also been reported as the second most preferred (38.36\%) type of treatment supporters in another cross-sectional study in Pakistan and a qualitative study in Nigeria noticed maximum emotional and physical support from the members of the family. The community-based treatment provider has been reported as the first choice (71.60\%) treatment provider in a mixed-method study in Uganda [811]. The present retrospective study revealed ASHAs as the most preferred treatment supporters by the patients, while family members were their second choice; a similar observation has also been made by Alipanah et al. [12].

The present study demonstrated that cure, treatment completion, death, treatment failure, or loss to followup were not affected by the type of treatment supporter but has an impact on transfer out. Another study has demonstrated that treatment outcome is not affected by the type of treatment supporter, so a patient should be allowed to choose a treatment supporter of choice [4].

A study conducted in Swaziland revealed the significant impact of treatment supporters on treatment outcomes as treatment completed, died, and transferred out but the significant impact was noticed on treatment outcomes as cured, treatment failed and defaulted. Some $60.90 \%$ having treatment supporters completed the treatment, $10.60 \%$ had died, and only $2.5 \%$ cases were transferred out. Some studies have demonstrated a significantly higher treatment success rate among DR-TB patients supported by treatment supporters. A cross-sectional survey in Rawalpindi revealed different success rates and transferred out cases with different types of treatment supporters. Treatment success rates with female health workers, community health workers, and family members recorded were $93.10 \%, 89.00 \%$, and $73.50 \%$ respectively, while transferred out cases noted were $2.0 \%, 00.00 \%$, and $3.20 \%$ respectively [13-16].

The present study revealed some impact of different types of treatment supporters on the treatment outcome. The significant impact of treatment supporters on treatment outcome as transferred out has been noted, while no significant impact of treatment supporters on treatment outcomes as cured, treatment completed, died, treatment failed, and lost to follow up has been demonstrated in the present study.

Transferred out occur when patients are not satisfied with the support provided by the treatment providers, Patients also try to migrate from their native place in Bihar to other states of India for jobs, business, and higher study. Counseling plays an important role in convincing them not to move outside the state till their treatment is completed. Some patients migrate to urban centers from rural ones with the hope to get better treatment facilities. It may also be due to the fact that treatment supporters start taking less interest in their work for not getting their honorarium in time [17].

\section{Conclusions}

The present study provides an assessment of different treatment supporters in the programmatic management of drug resistant tuberculosis (PMDT) program in India. This study highlights the role of different support providers and the impact of different types of treatment supporters on the treatment outcome of DR-TB patients. A significant impact of some treatment supporters is also evident in the treatment outcome. ASHAs seem to play a very important role as treatment supporters of DR-TB patients by decreasing or preventing the proportion of transfer out cases and this could be due to training that they have received and good counseling by them right from the start of the treatment.

\section{Additional Information}




\section{Disclosures}

Human subjects: Consent was obtained or waived by all participants in this study. Animal subjects: All authors have confirmed that this study did not involve animal subjects or tissue. Conflicts of interest: In compliance with the ICMJE uniform disclosure form, all authors declare the following: Payment/services info: All authors have declared that no financial support was received from any organization for the submitted work. Financial relationships: All authors have declared that they have no financial relationships at present or within the previous three years with any organizations that might have an interest in the submitted work. Other relationships: All authors have declared that there are no other relationships or activities that could appear to have influenced the submitted work.

\section{Acknowledgements}

We thank Mr. Manohar Mishra, Statistical Assistant NDR-TB Centre for statistical works. We also thank CDO and his team for their cooperation.

\section{References}

1. National TB Elimination Programme. Central TB Division, Ministry of Health and Family Welfare, Government of India, New Delhi: Guidelines for Programmatic Management of Drug Resistant Tuberculosis in India. 2021. 1-123.

2. World Health Organization: A Guide for Tuberculosis Treatment Supporters . WHO, Geneva; 2002. 1-20.

3. Nikshay Treatment Supporter Scheme (TSS), Ministry of Health and Family Welfare, Government of India: Nutritional Support to TB Patients (Nikshay Poshan Yojana) . 2022.

4. Singh A, Prasad R, Kushwaha RA, Srivastava R, Giridhar BH, Balasubramanian V, Jain A: Treatment outcome of multidrug-resistant tuberculosis with modified DOTS-plus strategy: A 2 years' experience. Lung India. 2019, 36:384-392. 10.4103/lungindia.lungindia_475_18

5. Pradipta IS, Van't Boveneind-Vrubleuskaya N, Akkerman OW, Alffenaar JC, Hak E: Treatment outcomes of drug-resistant tuberculosis in the Netherlands, 2005-2015. Antimicrob Resist Infect Control. 2019, 8:1-12. 10.1186/s13756-019-0561-z

6. Javaid A, Ullah I, Masud H, Basit A, Ahmad W, Butt ZA, Qasim M: Predictors of poor treatment outcomes in multidrug-resistant tuberculosis patients: a retrospective cohort study. Clin Microbiol Infect. 2018, 24:612617. 10.1016/j.cmi.2017.09.012

7. Hussain S, Hasnain J, Hussain Z, Badshah M, Siddique H, Fiske C, Pettit A: Type of treatment supporters in successful completion of tuberculosis treatment: a retrospective cohort study in Pakistan. Open Infect Dis J. 2018, 10:37-42. 10.2174/1874279301810010037

8. Izudi J, Tamwesigire IK, Bajunirwe F: Treatment supporters and level of health facility influence completion of sputum smear monitoring among tuberculosis patients in rural Uganda: a mixed-methods study. Int J Infect Dis. 2020, 91:149-155. 10.1016/j.ijid.2019.12.003

9. Mlilo N, Sandy C, Harries AD, et al.: Does the type of treatment supporter influence tuberculosis treatment outcomes in Zimbabwe?. Public Health Action. 2013, 3:146-148. 10.5588/pha.13.0002

10. Olukolade R, Hassan A, Ogbuji Q, et al.: Role of treatment supporters beyond monitoring daily drug intake for TB-patients: findings from a qualitative study in Nigeria. J Public Health. 2017, 9:65-73. 10.5897/JPHE2017.0909

11. Dave PV, Shah AN, Nimavat PB, et al.: Direct observation of treatment provided by a family member as compared to non-family member among children with new tuberculosis: a pragmatic, non-inferiority, cluster-randomized trial in Gujarat, India. PLoS One. 2016, 11:e0148488. 10.1371/journal.pone.0148488

12. Alipanah N, Jarlsberg L, Miller C, Linh NN, Falzon D, Jaramillo E, Nahid P: Adherence interventions and outcomes of tuberculosis treatment: a systematic review and meta-analysis of trials and observational studies. PLoS Med. 2018, 15:e1002595. 10.1371/journal.pmed.1002595

13. Kliner M, Mamvura C, Ndwandwe SZ, Busuwala F, Welfare W, Walley J, Wright J: Effects of financial incentives for the treatment outcome in Swaziland : a pragmatic interventional study. Infect Dis Poverty. 2015, 4:1-7.

14. Soomro MH, Qadeer E, Khan MA, Morkve O: Treatment supporters and their impact on treatment outcomes in routine tuberculosis program conditions in rawalpindi district, pakistan. Tanaffos. 2012, 11:15-22.

15. Wen S, Yin J, Sun Q: Impacts of social support on the treatment outcomes of drug-resistant tuberculosis: a systematic review and meta-analysis. BMJ Open. 2020, 10:e036985.

16. Bhatt R, Chopra K, Vashisht R: Impact of integrated psycho-socio-economic support on treatment outcome in drug resistant tuberculosis - a retrospective cohort study. Indian J Tuberc. 2019, 66:105-110. 10.1016/j.ijtb.2018.05.020

17. Belayneh T, Kassu A, Tigabu D, Asmare G, Tilaye S, Klinkenberg E: Characteristics and treatment outcomes of "Transfer-Out" pulmonary tuberculosis patients in Gondar, Ethiopia. Tuberc Res Treat. 2016, 2016:1294876. 10.1155/2016/1294876 